\title{
Squamous Cell Carcinoma of the External Ear in a Child
}

\author{
Waheed Rahman, Rashid Sheikh*, Hassen Mohammed, Zeynel Abidin Dogan \\ Department of Otorhinolaryngology and Head \& Neck Surgery, Hamad Medical Corporation, Doha, Qatar \\ Email: rsheikh@hamad.qa
}

Received 7 July 2015; accepted 28 August 2015; published 31 August 2015

Copyright (C) 2015 by authors and Scientific Research Publishing Inc.

This work is licensed under the Creative Commons Attribution International License (CC BY). http://creativecommons.org/licenses/by/4.0/

c) (i) Open Access

\begin{abstract}
Tumors involving the head and neck are uncommon in children. Furthermore, those which involve the external ear are extremely rare. In the external ear itself, the most commonly encountered malignancy is Squamous Cell Carcinoma, both in the adult and pediatric age groups. We encountered one such case of a 14 years old male with a recurring skin lesion involving the right external ear. In this report, we wish to highlight and address the difficulty in recognizing and managing such an unwonted pathology as Squamous Cell Carcinoma in the pediatric age group.
\end{abstract}

\section{Keywords}

Squamous Cell Carcinoma, External Ear, External Auditory Canal

\section{Introduction}

Squamous cell carcinoma (SCC) is a common malignancy arising from malignant proliferation of the keratinocytes of the epidermis. It is primarily a disease of older adults, occurring most frequently in patients older than age 45 years. Epidemiological studies over the last 20 years have shown a steady rise in the incidence of these cancers in younger adults [1].

Many reports had been published on the cause(s), risk factors, progression and prognosis of SCC in young adults since this disease was previously recognized as a distinct clinical entity, but they were inconsistent. Younger patients often do not present with the conventional risk factors of alcohol and/or tobacco exposure. This leads to a suspicion that other potential agents, such as genetic factors, infections, and behavioral factors may be involved [2]-[4].

Furthermore, if SCC involves the head and neck region, in particular the external ear, it can be potentially lethal. Therefore, managing such a case requires an early and aggressive interventionist approach. In this report,

\footnotetext{
*Corresponding author.
}

How to cite this paper: Rahman, W., Sheikh, R., Mohammed, H. and Dogan, Z.A. (2015) Squamous Cell Carcinoma of the External Ear in a Child. International Journal of Otolaryngology and Head \& Neck Surgery, 4, 338-343. 
we focus on the presentation, diagnosis, existing literature and the tailored management of such a rare pathology in a 14 years old male.

\section{The Case}

A 14 year old male presented with an isolated, rapidly recurring, painless, raised, hyperpigmented and ulcerated lesion which was approximately $2 \mathrm{~cm} \times 2 \mathrm{~cm}$ in size on his right tragus involving the preauricular skin and the external auditory canal (EAC) (Figure 1). It was increasing in size over the preceding 3 months. The patient underwent excision of a lesion in the same location 6 months before presenting to our Otolaryngology service. He had also undergone 5 prior excisions of similar lesions in the same location. The child's parents had lost the records of most of the histopathology results. The available results of 3 out of 6 presumed excisional biopsies were: pyogenic granuloma, atypical dysplasia and basal cell carcinoma.

Informed verbal and written consents were taken from the parents. Radiological imaging ensued (Figure 2). It was decided to proceed with excisional biopsy of the lesion under general anesthesia. Subsequent histopathology result showed moderately differentiated SCC that involved the tragus, preauricular skin and external auditory canal. The tumor was invading the cartilagenous part of the right EAC encroaching upon the parotid capsule without gross invasion. Medially the tumor extended to the skin of the bony EAC without gross erosion of the bone.

A detailed discussion in the multidisciplinary tumor board meeting occurred prior to initiating definitive management. High risk features in this case included recurrence, location and cartilage invasion. Eventually, with a therapeutic and curative intent, subtotal right external ear canal excision with reconstruction along with right supraomohyoid neck dissection and right total parotidectomy was done with tumor free margins on frozen section and final histopathology.

Microscopic examination of the sections revealed the tumor to be residual invasive carcinoma with squamous differentiation. Scar and changes consistent with previous biopsy site were noted. It was unifocal with $1.0 \mathrm{~cm}$ in greatest dimension with no adjacent squamous dysplasia or carcinoma in-situ. Histologic grade was poorly differentiated. It was adjacent to the parotid gland with invasion of the capsule and surrounding soft tissue while bone and cartilage was spared. Margins were free of the tumor. While tumor necrosis was focally present, there was no lymphovascular or perineural invasion. The tumor consisted of sheets, islands and trabeculae of abnormal squamous cells showing pleomorphism, nucleomegaly and frequent mitotic figures (Figure 3). The cells showed cytoplasmic keratinization and focal squamous pearls (Figure 4). The tumor infiltrated the subepithelial stroma where it extended to the cartilaginous plate. Immuohistochemical staining showed positive staining with CK5/6, CK14, AE1/AE3, p63 and EMA (antibodies that support squamous nature for the tumor) (Figure 5 \& Figure 6). The tumor cells were negative with CK7, CK20, HPV, and EBV. Ki-67 index > 50\%. Insitu hybridization was performed using special antibody against EBV (EBER antibody). There was no staining with the antibody. Appropriate positive and negative controls were working. The pathologic stage (pTNM) was verified to be was PT1, N0.

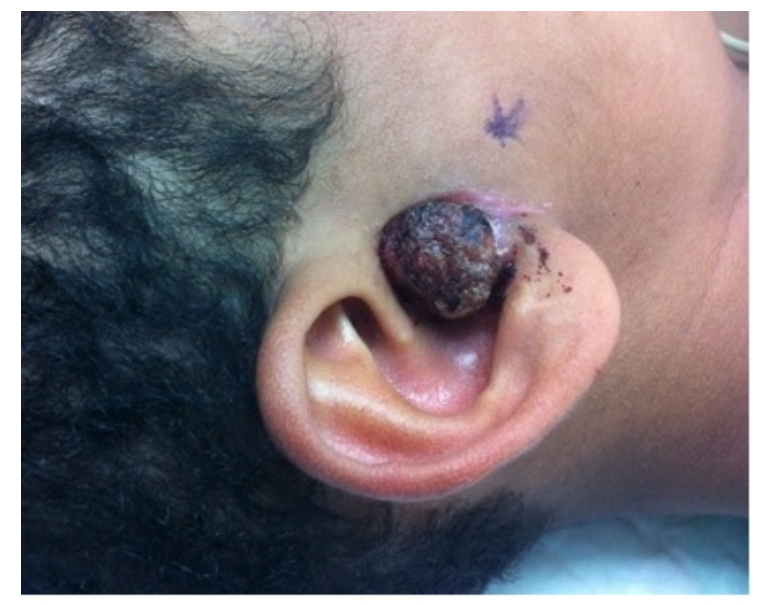

Figure 1. Appearance of the lesion at presentation. 


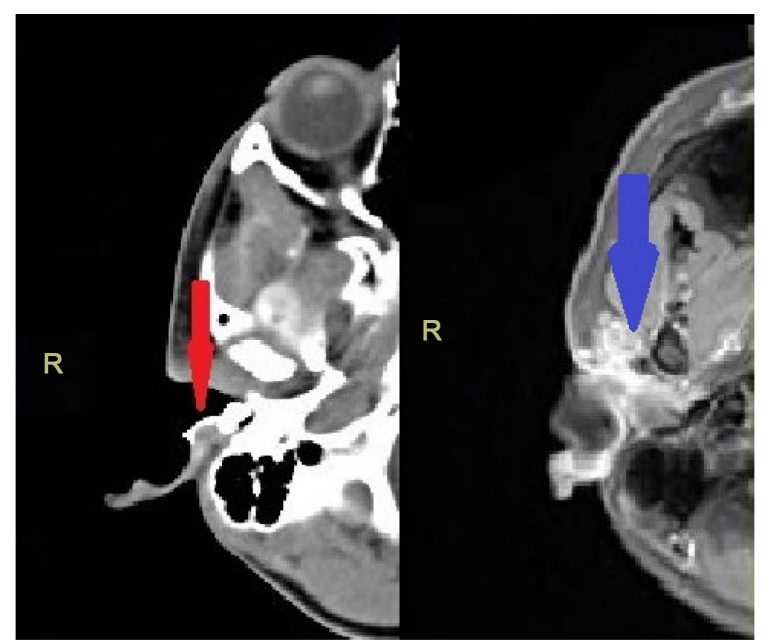

Figure 2. Imaging of the right side of the head. CT Scan showing the lesion invading the external ear canal (red arrow) and MRI showing the same lesion (blue arrow).

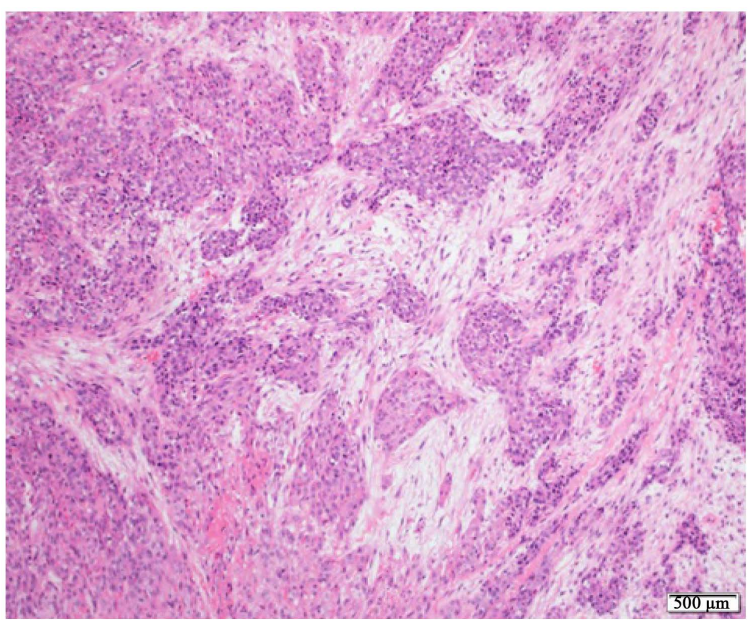

Figure 3. Sections show islands and sheets of tumor cells invading the stroma (H \& E $\times 100)$.

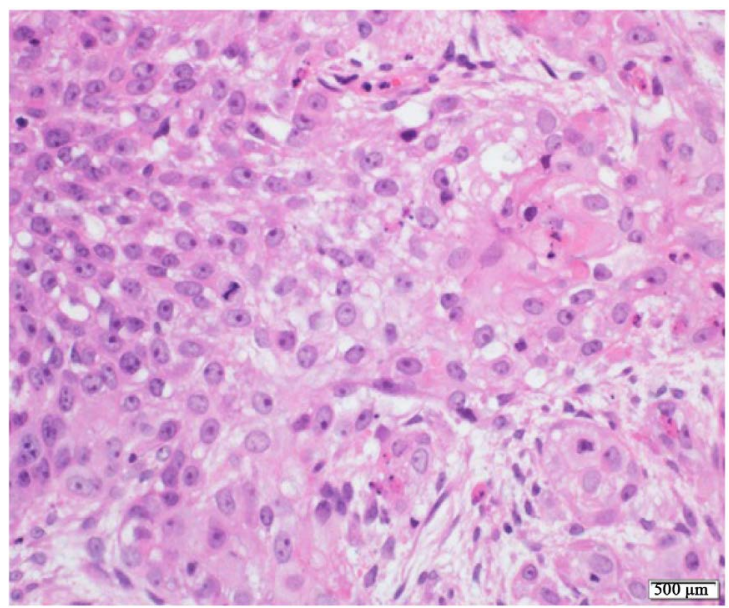

Figure 4. High power view showing the atypical squamous cells with cytoplasmic keratinization (right side of slide) (H \& E ×400). 


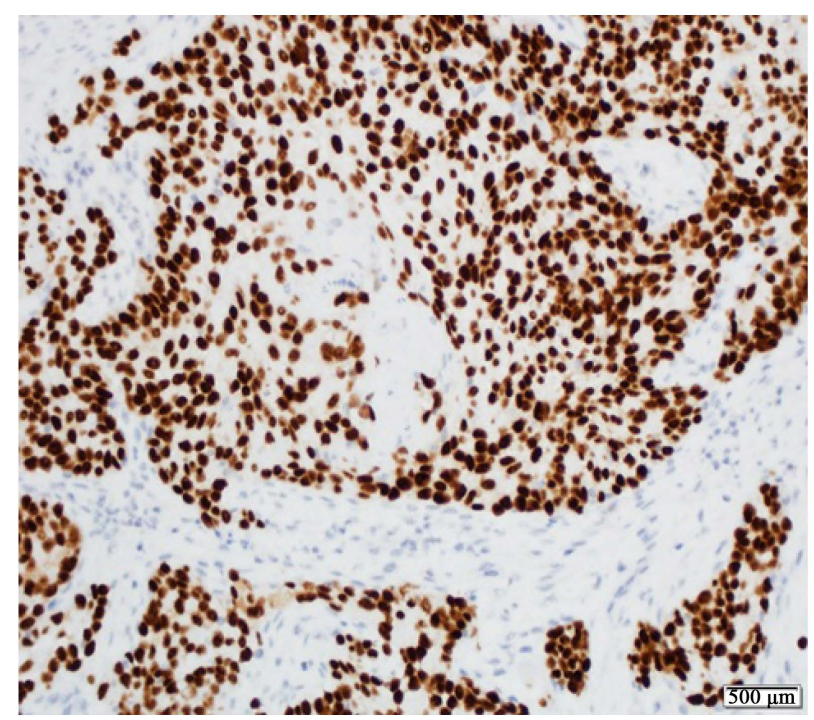

Figure 5. Diffuse nuclear staining with p63 antibody (immuoperoxidase $\times 200$ ).

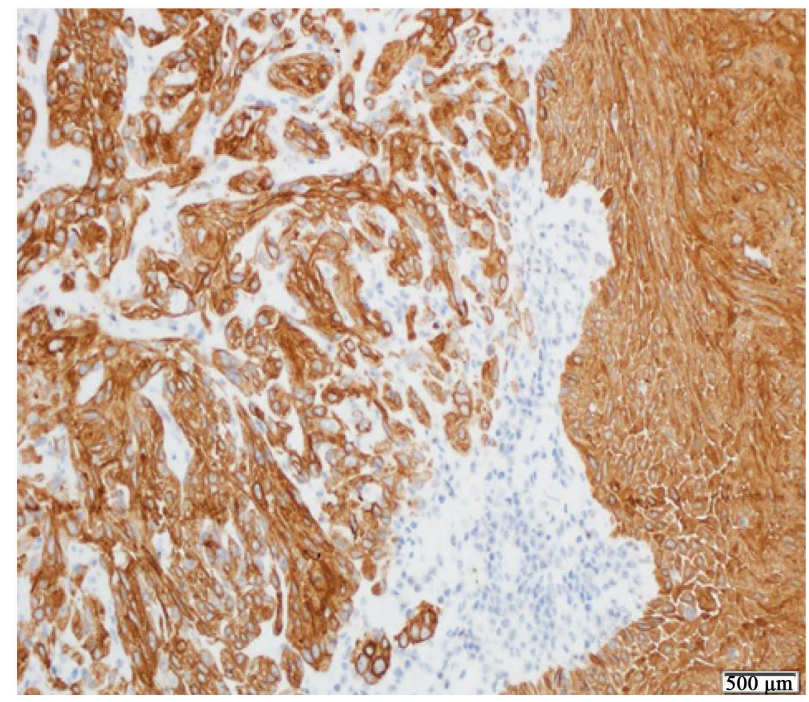

Figure 6. Diffuse membranous and cytoplasmic staining with CK14 antibody $\times 200$.

To date and a year on from the definitive surgery, the child has had no signs of local or distant recurrence of the tumor and the surgical wound has healed well. Two of the patient's six monthly followup radiology surveillance imaging reports suggested no evidence of local or distant recurrence.

\section{Discussion}

SCC of the head and neck region is unusual in young patients and extremely rare in the pediatric age group, the upper limit of which varies in different centers let alone countries and regions [5]. SCC of the head and neck is a disease of middle age and beyond usually in patients who are exposed to its primary risk factors which are smoking and alcohol consumption. A portion of patients who are younger, however, develop SCC of the head and neck without the use of tobacco or alcohol and it is this population in which the etiology of their cancer comes into question. Debate regarding the tumor nature in this group of patients and its effects on progression and survival is open-ended [6]. Like most cancers, there isn't a single etiology and it has been determined that multiple factors may be involved.

EAC carcinomas, let alone SCC as in this case, are frequently misdiagnosed. A retrospective study to review 
misdiagnosed cases and analyze the factors involved was done by Zhang T. et al. [7]. Eighteen of 44 EAC carcinoma cases seen were misdiagnosed: Six as otitis media, five as otitis externa and two as EAC cholesteatomas. Other misdiagnoses were stenosis of the EAC, ear neuralgia, furuncle of the EAC, benign neoplasm of the EAC and pre-auricular fistula. Our case stands unique as the clinical presentation was of a recurring lesion despite excisional biopsies and at the same time histopathology results differed repeatedly. No particular reason, apart from the age of the patient, was outlined to explain this. It leads us to believe that the pathologists were not cautious in making the diagnosis as the microscopic description did not provide any clue as to why the diagnosis was missed.

Young patients with SCC reported in literature are isolated cases and their management is difficult because there is no large study to support treatment decision for every child [5]. A CT scan and/or MRI of the head and neck is recommended to note bony erosion and/or soft tissue involvement of a suspected tumor of the EAC. A low threshold of suspicion for possible underlying malignancy should exist for otitis media or externa cases, especially those with bloody ear discharge and/or otalgia, which do not respond to routine anti-bacterials. A prompt biopsy should be conducted to obtain a histopathological diagnosis [7] [8].

Upto 75 cases of SCC of the external ear were reviewed to determine patterns of occurrence and treatment failure in a series by Shockley et al. Forty patients had adequate follow-up for determination of cancer control rates. Local control was successful with initial treatment in $85 \%$ of the cases. The incidence of lymph node metastases was 10\%, whereas distant metastasis occurred in only one patient (2.5\%) [9]. It has been observed that young patients with SCC of the head and neck have an analogous prognosis to older patients. However, a higher regional recurrence in young patients has been noted in recent times which has prompted surgeons to utilize prophylactic neck dissection to halt the unusual rise in recurrence [6]. Adding to that, Federspil et al. showed that tumor excision with wide margins $(5-10 \mathrm{~mm})$ as first-line treatment is recommended. He also noted that neck dissection with parotidectomy is indicated when suspicious lymph nodes are detected, the tumor diameter is $>4 \mathrm{~cm}$, cartilaginous invasion is present, and vertical tumor thickness is $>5 \mathrm{~mm}$ [10].

Keeping in view existing knowledge of the management of such a case, it is pertinent and worthwhile to mention that the management of SCC of the skin differs from SCC of the external auditory canal. In this case, repeated surgical intervention had obscured the path of progression of the disease, whether it was from the skin to the external ear canal or vis versa. We approached the case as an EAC SCC which propelled us to take a more aggressive approach. Another dilemma which we encountered was whether to give the patient adjuvant radiotherapy or not. Thus far, after following the patient for over 12 months, there are no signs of recurrence and the patient has not received radiotherapy. We can also safely assume that if the margins of the excised tumor are clear, then such cases may not merit use of radiotherapy and can be considered tumor free or in remission.

\section{Conclusion}

This case has been reported to sensitize the reader, the histopathologist and the otorhinolaryngologist and head \& neck surgeon that the possibility of Squamous Cell Carcinoma should be high on their differential list even in the pediatric age group. Misdiagnosis may lead both the patient and doctor off track with regards to the best course of management of the disease especially in cases where intervention is indicated. Tools of diagnosis and staging including imaging and histopathology should be utilized effectively prior to commencing on the management of such a disease. In order to prevent recurrence, a more radical and aggressive approach should be taken which has been seen to cause remission of the disease without utilizing adjuvant radiotherapy.

\section{Disclosure}

The case at hand has already been approved by our institution's medical research and ethics committee at the research center with the reference number of MRC0575/2015. The patient's father's consent was taken for the publication of the case report and the figures. None of the authors was given an honorarium, grant or other form of payment to produce this paper. We have no conflict of interest or financial interest, real or perceived, to disclose.

\section{References}

[1] Chaturvedi, A.K., Engels, E.A., Anderson, W.F. and Gillison, M.L. (2008) Incidence Trends for Human Papillomavirus-Related and -Unrelated Oral Squamous Cell Carcinomas in the United States. Journal of Clinical Oncology, 26, 612-619. http://dx.doi.org/10.1200/JCO.2007.14.1713 
[2] Myers, J.N., Elkins, T., Roberts, D. and Byers, R.M. (2000) Squamous Cell Carcinoma of the Tongue in Young Adults: Increasing Incidence and Factors that Predict Treatment Outcomes. Otolaryngology-Head and Neck Surgery, 122, 44-51. http://dx.doi.org/10.1016/S0194-5998(00)70142-2

[3] Sturgis, E.M. and Cinciripini, P.M. (2007) Trends in Head and Neck Cancer Incidence in Relation to Smoking Prevalence. Cancer, 110, 1429-1435. http://dx.doi.org/10.1002/cncr.22963

[4] Byers, R.M. (1975) Squamous Cell Carcinoma of the Oral Tongue in Patients Less than Thirty Years of Age. The American Journal of Surgery, 130, 475-478. http://dx.doi.org/10.1016/0002-9610(75)90487-0

[5] de Carvalho, M.B., de Andrade Sobrinho, J., Rapoport, A., et al. (1998) Head and Neck Squamous Cell Carcinoma in Childhood. Medical and Pediatric Oncology, 31, 96-99.

http://dx.doi.org/10.1002/(SICI)1096-911X(199808)31:2<96::AID-MPO9>3.0.CO;2-U

[6] Goldstein, D.P. and Irish, J.C. (2005) Head and Neck Squamous Cell Carcinoma in the Young Patient. Current Opinion in Otolaryngology \& Head and Neck Surgery, 13, 207-211. http://dx.doi.org/10.1097/01.moo.0000170529.04759.4c

[7] Zhang, T., Dai, C. and Wang, Z. (2013) The Misdiagnosis of External Auditory Canal Carcinoma. European Archives of Oto-Rhino-Laryngology, 270, 1607-1613. http://dx.doi.org/10.1007/s00405-012-2159-4

[8] Hosokawa, S., Mizuta, K., Takahashi, G., et al. (2012) Surgical Approach for Treatment of Carcinoma of the Anterior Wall of the External Auditory Canal. Otology \& Neurotology, 33, 450-454. http://dx.doi.org/10.1097/MAO.0b013e318245ccbf

[9] Shockley, W.W. and Stucker Jr., F.J. (1987) Squamous Cell Carcinoma of the External Ear: A Review of 75 Cases. Otolaryngology—Head and Neck Surgery, 97, 308-312.

[10] Federspil, P.A., Pauli, U.C. and Federspil, P. (2001) Squamous Epithelial Carcinomas of the External Ear. HNO, 49, 283-288. http://dx.doi.org/10.1007/s001060050747 